\title{
A Study of Multi-Layer Spiral Inductors for Remote Powering of Implantable Sensors
}

\author{
Jacopo Olivo, Sandro Carrara, Member, IEEE, and Giovanni De Micheli, Fellow, IEEE
}

\begin{abstract}
An approach based on multi-layer spiral inductors to remotely power implantable sensors is investigated. As compared to single-layer inductors having the same area, multi-layer printed inductors enable a higher efficiency (up to $35 \%$ higher) and voltage gain (almost one order of magnitude higher). A system conceived to be embedded into a skin patch is designed to verify the performance. The system is able to transmit up to $15 \mathrm{~mW}$ over a distance of $6 \mathrm{~mm}$ and up to $1.17 \mathrm{~mW}$ where a $17 \mathrm{~mm}$ beef sirloin is placed between the inductors. Furthermore, the system performs downlink communication (up to $100 \mathrm{kbps}$ ) and uplink communication based on the backscattering technique (up to $66.6 \mathrm{kbps}$ ). Long-range communication is achieved by means of a bluetooth module.
\end{abstract}

Index Terms-Energy harvesting, implantable sensors, inductive link, multi-layer spiral inductors, remote powering.

\section{INTRODUCTION}

$\mathbf{T}$ HE research field of implantable biosensors has been attracting the attention of academia and industry for the past two decades. The possibility to constantly monitor the human body and its metabolism from inside paves the way to personalized medicine and point-of-care therapies, giving the opportunity to adapt the medical treatments to the response of the patient. For example, the constant monitoring of the glucose level in the subcutaneous interstitial fluids is an important aid to those patients who suffer from diabetes. By means of implantable sensors located in the subcutaneous zones, the percentage of glucose into the blood (glycemia) can be recorded and transmitted to a remote device, such as a smartphone, to be analyzed. Thus, periodic and disturbing blood drawing can be avoided. Another metabolite that can be monitored by means of subcutaneous sensors is lactate, a product of the anaerobic muscle activity [1]. The lactate concentration into the blood (lactatemia) or into interstitial tissues in muscles can be recorded to monitor the muscular effort in sportsmen or people under rehabilitation.

\section{A. Remote Powering of Lactate Biosensors}

Different solutions have been proposed in literature to design and optimize amperometric biosensors dedicated to the lactate

Manuscript received February 07, 2012; revised May 19, 2012 and August 10, 2012; accepted September 28, 2012. Date of publication January 04, 2013; date of current version July 24, 2013. This work was supported by the i-IronIC project and by the NanoSys project. The NanoSys project is within the program ERC-2009-AdG-246810. The i-IronIC project was supported by a grant from the Swiss Nano-Tera.ch initiative and evaluated by the Swiss National Science Foundation. This paper was recommended by Associate Editor M. Stanacevic.

The authors are with the Integrated Circuits Laboratory, EPFL - École Polytechnique Fédérale de Lausanne, 1015 Lausanne, Switzerland (e-mail: jacopo. olivo@epfl.ch; sandro.carrara@epfl.ch; giovanni.demicheli@epfl.ch).

Color versions of one or more of the figures in this paper are available online at http://ieeexplore.ieee.org.

Digital Object Identifier 10.1109/TBCAS.2012.2225620 detection [2], [3]. The use of carbon nanotubes has been proposed to enhance the sensitivity of these devices [4], [5]. The presence of nanostructured electrodes slightly complicates the design of the sensor. Due to the toxicity of nanotubes [6], [7], selective membranes must be used to avoid dispersion into the body. However, the enhanced sensitivity enables the detection of small concentrations of the metabolite, typical of the body range.

Besides the sensing aspects, related to the electrochemistry domain, implantable biosensors must respect several constraints. They must be minimally invasive, completely biocompatible, with low thermal dissipation, and large power autonomy. This last requirement should be carefully considered, since battery size and battery lifetime strongly affect the performance and the invasivity of an implantable sensor. Modern batteries, even with an improved energy density, still represent a bottleneck in the miniaturization of the implantable sensors. Moreover, substituting the battery can cause discomfort to the patient.

Energy harvesting techniques exploit natural or artificial power sources surrounding the person to assist the implanted batteries and in certain cases replace them. Previously developed harvesting techniques for implantable biosensors are surveyed in [8]. Remote powering through inductive links is one of the most promising approaches. In such a technique, near-field magnetic induction is used to transfer power wirelessly through the body tissues. An alternate current is forced into an external inductor; the variable magnetic field generated induces an alternate current into one or more receiving inductors. This technique has been studied since several decades, and it has already reached the market with commercial products [9]. However, the miniaturization process of the receiving inductors, while preserving power efficiency, is still an open research topic.

This article explores the use of multi-layer printed spiral inductors to remotely power subcutaneous devices dedicated to lactate monitoring. Thanks to this approach, the size of the receiver inductors can be noticeably reduced with respect to the classical "pancake" inductors or the single-layer spiral inductors, such as the ones used in [10]-[15]. Moreover, the decrease in size does not result in a lower efficiency of the system in terms of power transmission. Finally, the small size of the multi-layer spiral inductors supports their implantation in the subcutaneous zones by means of an injection.

The proposed approach is initially compared to different solutions presented in literature, such as litz-wire coils, on-chip inductors, and MEMS inductors. Then, the advantages of using multi-layer structures, in terms of link efficiency and voltage gain, are investigated and compared with a single-layer approach. 


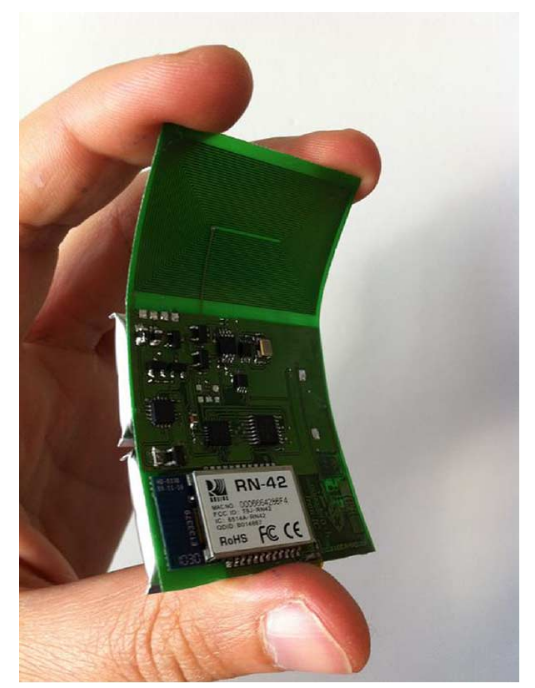

Fig. 1. A system is designed to power implantable sensors through an inductive link. The system is realized on a flexible substrate to be embedded into a skin patch and is used to test multi-layer receiving inductors. Reprinted from [16].

A system, shown in Fig. 1, is designed to confirm the benefits of using multi-layer spiral inductors [16]. It utilizes a high efficiency class-E power amplifier to drive the external inductor. Bidirectional data communication between the system and the implantable device is performed. Downlink communication (from the external system to the implantable sensor) is achieved by means of an external amplitude modulator (Amplitude Shift Keying-ASK). Uplink communication (from the implantable sensor to the external system) is obtained by varying the internal load (Load Shift Keying-LSK) and detecting this change on the current flowing on the external inductor. Thus, no implantable RF transmitter is used. This technique is usually named backscattering. In addition to the short-range communication with the implantable sensor, the system performs long-range communication with a remote device by means of an embedded bluetooth module. Indeed, the system is designed to enable real-time telemetry of the body metabolism.

The realization of the external inductor on a flexible substrate, such as a polyamide film, increases the wearability of the device that can be embedded into skin patches and located directly over the implantation areas (Fig. 1). The improved wearability, when compared to systems using classical "pancake" inductors as external coils [10], [11], [13], reduces the discomfort of the patient.

The remainder of the paper is organized as follows. Section II introduces proper figures of merit used to evaluate the quality and the performance of an inductive link. In Section III different techniques to realize an inductive link are presented and compared. Moreover, the multi-layer approach is introduced. Section IV describes the design of the link by using multi-layer printed spiral inductors. Simulations and measurements are used to determine the inductor geometry and the number of layers. Section $\mathrm{V}$ reports a brief description of the external transmitter and the experimental results. Finally, Section VI concludes the paper.

\section{Figures OF MERIT OF AN INDUCTIVE LINK}

An inductive link is an electrical circuit consisting of two or more magnetically-coupled inductors. In the case of remote

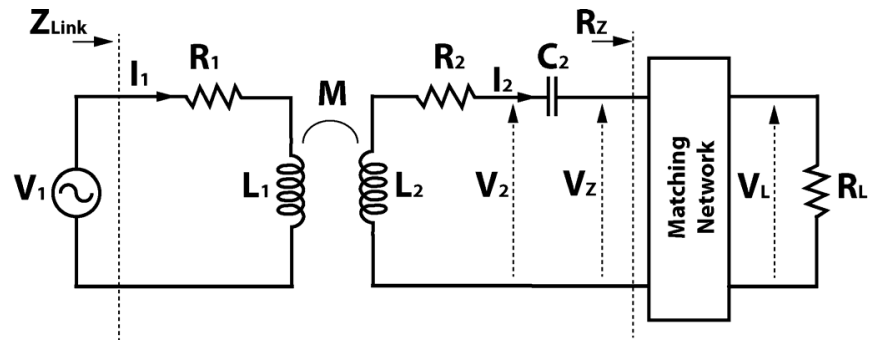

Fig. 2. Schematic description of an inductive link.

powering of implanted biosensors, the link usually consists of two inductors. The external (transmitting) inductor is placed outside the body, while the internal (receiving) inductor is implanted. An alternate current flowing on the external inductor changes the magnetic flux linked to the internal inductor. Thus, an electromotive force is generated at the edges of the internal inductor. If the internal inductor is connected to a load, a current flows through the load.

An inductive link with two inductors is shown in Fig. 2. The external inductor $L_{1}$, drawn with its equivalent series resistance $R_{1}$, is driven by the voltage source $V_{1}$. The internal inductor $L_{2}$ is also drawn with its equivalent series resistance $R_{2}$. By making the assumption that the link operates fairly below the self-resonance frequencies of the two inductors, no other intrinsic component has to be reported in the schematic.

A resonant capacitor $C_{2}$ is used to drive the secondary circuit into resonance and maximize the power transfer and the link efficiency [17]. In order to resonate with the inductor $L_{2}$ at the working angular frequency $\omega$, the value of $C_{2}$ is fixed at $C_{2}=1 / \omega^{2} L_{2}$. Finally, a passive matching network is used to achieve impedance matching between the coil resistance $R_{2}$ and the load $R_{L}$. Typically, a pure capacitive network can be used to match the impedance of the load with the receiving inductor. If the parasitic resistances of those capacitors are neglected, the matching network does not dissipate active power. This ideal behavior is assumed throughout the paper.

\section{A. Link Efficiency}

The efficiency $\eta$ of an inductive link is defined as the ratio between the power dissipated on the load and the total dissipated power. We report here the analytical formulas presented in [17] to describe the link efficiency $\eta$ as a function of the electrical parameters of the link. By defining $\eta_{1}$ as the power dissipated on the secondary circuit over the totally dissipated power

$$
\eta_{1}=\frac{\omega^{2} M^{2}}{\omega^{2} M^{2}+R_{1}\left(R_{2}+R_{Z}\right)}
$$

and $\eta_{2}$ as the power dissipated on the load over the power dissipated on the secondary circuit

$$
\eta_{2}=\frac{R_{Z}}{R_{Z}+R_{2}}
$$

the link efficiency $\eta$ can be written as

$$
\eta=\eta_{1} \eta_{2}=\frac{\omega^{2} M^{2} R_{Z}}{\omega^{2} M^{2}\left(R_{2}+R_{Z}\right)+R_{1}\left(R_{2}+R_{Z}\right)^{2}} .
$$


By defining the coupling coefficient $k$ as

$$
k=\frac{M}{\sqrt{L_{1} L_{2}}}
$$

and the quality factor $Q$ as

$$
Q=\frac{\omega L}{R}
$$

the efficiency reported in (3) can be rewritten as

$$
\eta=\frac{k^{2} Q_{1} Q_{2} \frac{R_{2}}{R_{Z}}}{\left(1+\frac{R_{2}}{R_{Z}}+k^{2} Q_{1} Q_{2} \frac{R_{2}}{R_{Z}}\right)\left(1+\frac{R_{2}}{R_{Z}}\right)} .
$$

The ratio between the intrinsic resistance $R_{2}$ of the internal inductor and the load $R_{Z}$ that maximizes the link efficiency is obtained

$$
\frac{\partial \eta}{\partial R_{Z}}=0 \Longrightarrow R_{Z}=R_{2} \sqrt{1+k^{2} Q_{1} Q_{2}}
$$

Since

$$
\left.\frac{\partial^{2} \eta}{\partial R_{Z}^{2}}\right|_{R_{Z}=R_{2} \sqrt{1+k^{2} Q_{1} Q_{2}}}<0
$$

the stationary point found in (7) is a maximum.

In the remainder of the paper, $R_{Z}$ is chosen according to $R_{2}$ to satisfy the expression (7). The desired $R_{Z}$ is obtained by properly tuning the matching network.

Finally, by combining (3) and (7), the maximum link efficiency is described by

$$
\eta_{\max }=\frac{k^{2} Q_{1} Q_{2}}{\left(1+\sqrt{1+k^{2} Q_{1} Q_{2}}\right)^{2}} .
$$

\section{B. Voltage Gain}

The transferred power, although important, is not the only figure of merit of an inductive link. In order to power a load, the supplied voltage must be sufficiently high, according to the load specification. Charge pumps can be used to increase the voltage supplied to the load; however, the voltage at the input of the charge pumps must be sufficient to activate them.

Thus, it is useful to define the voltage gain $A$ as the ratio between the voltage $V_{Z}$ and the voltage of the source $V_{1}$. According to [17], the voltage gain $A$ can be written as

$$
A=\frac{\left|V_{Z}\right|}{\left|V_{1}\right|}=\frac{\omega M R_{Z}}{\sqrt{\left[R_{1}\left(R_{2}+R_{Z}\right)+\omega^{2} M^{2}\right]^{2}+\omega^{2} L_{1}^{2}\left(R_{2}+R_{Z}\right)^{2}}} .
$$

By studying the voltage gain $A$ as a function of the load $R_{Z}$, no maximum is found for finite values of $R_{Z}$. The voltage gain asymptotically tends to a maximum as $R_{Z}$ tends to infinity (open circuit). As previously stated, in the remainder of the paper the value of $R_{Z}$ that maximizes the link efficiency is considered as reported in (7), even if this value does not maximize the voltage gain.

\section{Design Techniques For Inductive LinKS}

The design of the inductors composing the link greatly affects both the performance of the system and the comfort of the patient. Indeed, the small size of the receiving inductor noticeably simplifies the implantation process and reduces the discomfort of the patient. Litz-wire coils, integrated CMOS inductors, MEMS inductors, and printed spiral coils are the most common solutions for the receiving inductors.

Litz wires consist of many thin strands, individually insulated and twisted or woven together, according to a predefined pattern. Then, those wires can be used to obtain the receiving coil (litz-wire coils).

Litz-wire inductors have already been used for the remote powering of implantable systems [12], [18]. This approach reduces the resistive losses due to the skin and proximity effects [19]. However, the advantages of using litz wires, rather than solid wires, decrease for frequencies higher than hundreds of kilohertz. By increasing the frequency over that range, a threshold is reached where local proximity losses increase and litz wires become more lossy than solid wires. This threshold has been estimated close to $1 \mathrm{MHz}$ [20]. Usually, inductive links for the remote powering of implantable devices operate in a slightly higher frequency range, between $1 \mathrm{MHz}$ and 13.56 MHz, with two dedicated frequencies at $6.78 \mathrm{MHz}$ and 13.56 MHz [10]-[15], [21], [22]. Thus, frequency limitations are a drawback of using litz-wire coils for the remote powering of implantable sensors.

Frequency issues can be mitigated by increasing the number of strands, while reducing the diameter. However, physical limits exist on strand reduction. Moreover, a smaller diameter of the strands results in a decreased packing factor, defined as the ratio between the copper area and the area of the bundle [20].

Another implementation is the integration of spiral inductors on-chip with the logic circuitry to be powered by the receiver. This approach considerably reduces the area of the system. However, by using a standard CMOS fabrication process, passive inductors usually have small inductance (typically in the range of nanohenries [23]) and high resistance, leading to quality factors with maximum value at high frequency (from hundreds megahertz up to few gigahertz) and usually lower than 10 [24], [25]. For these reasons, this approach is not suitable for power links operating in the low megahertz range.

These limitations can be surmounted by using different micro-fabrication techniques. Several solutions have been presented in literature employing MEMS inductors. By using thick-metal surface micromachining technology, [26] significantly decreased the inductor resistance, as compared to standard CMOS inductors. This decrease enables higher quality factors. However, since the inductance values remain in the order of few nanohenries, those quality factors still have their maximum in the gigahertz range.

Electroplating techniques can be used to obtain microfabricated inductors with lower resistance, when compared to thin film inductors. An inlaid electroplating procedure is used in [27] 
to realize microcoils on silicon substrates. The fabricated inductor has an area of $14 \times 14 \mathrm{~mm}^{2}$ and a quality factor of about 34 at $4 \mathrm{MHz}$. Similar performance is obtained by using electroplated gold on an insulating substrate [28]. The fabricated inductor has an area of $15 \times 15 \mathrm{~mm}^{2}$ and a quality factor of 29 at 13.56 MHz.

Finally, another option to design the receiving inductor is to use a standard Printed Circuit Board (PCB) process. This method enables the realization of the receiving coil on rigid or flexible substrates. Flexible substrates can considerably facilitate the implantation process. Furthermore, by using printed inductors several layers can be stacked to obtain multi-layer structures. The multi-layer approach enables area reduction, while preserving the performance of the link. Differently from what happens with microfabrication, no technological limitations exist on the number of layers that can be stacked.

The multi-layer approach has already been presented in literature [29]-[31]. Ref. [30] has proposed a $10 \times 10 \mathrm{~mm}^{2}$, 4-layer, $2.5 \mu \mathrm{H}$ receiving inductor having a quality factor of 90 at $13.56 \mathrm{MHz}$. These results are obtained by simulations. The effects on the inductance and the quality factor of different parameters (e.g., number of layers, spacing between adjacent traces, and trace width) are reported. A set of equations is proposed to calculate the electrical parameters of multi-layer structures starting from their physical characteristics [31]. A $9 \times 9 \mathrm{~mm}^{2}$, 2-layer, $3.8 \mu \mathrm{H}$ receiving inductor with a quality factor of 42 at $10 \mathrm{MHz}$ has been developed by using these analytic expressions [31].

Several key points make this technique particularly suitable for the remote powering of implantable systems. The implantation process can be facilitated by the flexibility of the substrate and the possibility to partially reduce the inductor area, while preserving the link performance. Moreover, a relatively high quality factor can be obtained with a simple and inexpensive process.

The drawbacks of this technique are the loss of flexibility when a high number of layers is used, the process resolution that limits the minimum size of the coil and the brittleness of the solder joints while dealing with a high number of layers. These constraints are determined by the technology and cannot be overcome. However, a careful design of the inductors (limited number of layers, careful choice of the inductor geometries and of the solder joints) can limit the effects of these drawbacks.

This paper further investigates the use of multi-layer inductors for the remote powering of implantable systems. A novel, asymmetrical shape of the receiving inductor is investigated. Due to a rectangular shape, having the form factor of a needle, the implantation process can be facilitated. This reduces the discomfort of the patient. Multi-layer rectangular structures are realized on a flexible substrate and measured, in order to compare their performance with the simulations reported in literature. Then, the concept of multi-layer inductors is deepened by studying their performance in the presence of biological tissues. Finally, a complete system is presented to perform measurements within an experimental setup.

\section{Design of Multi-Layer Inductors}

In this section the design of multi-layer printed spiral inductors is discussed. The electrical properties of the body tissues are determined for different implantation areas [32] (Section IV-A). Those data are used to simulate the effects of the inductor geometry on the optimal working frequency [32] (Section IV-B). Once a range of working frequencies is defined, the effects of the geometry on the link efficiency and the voltage gain are measured (Section IV-C). Finally, multi-layer inductors are introduced and measured (Section IV-D).

\section{A. Electrical Properties of the Body Tissues}

In order to investigate the behavior of an inductive link that remotely powers an implantable device, it is necessary to model the electrical properties of the appropriate human tissues. A large number of tissues have been investigated with the ColeCole dispersion model [33]. This model describes the complex permittivity $\hat{\varepsilon}$ of a tissue as a function of the angular frequency $\omega$ of the incident electromagnetic field

$$
\hat{\varepsilon}=\varepsilon_{\infty}+\sum_{n} \frac{\Delta \varepsilon_{n}}{1+\left(j \omega \tau_{n}\right)^{\left(1-\alpha_{n}\right)}}+\frac{\sigma_{i}}{j \omega \varepsilon_{0}}
$$

where $\tau$ is a time constant characterizing the polarization mechanism, $\alpha$ is a measure of the broadening of the dispersion, $\varepsilon_{0}$ is the permittivity of the vacuum and $\sigma_{i}$ is the static ionic conductivity of the tissue. The term $\varepsilon_{\infty}$ is the permittivity of the tissue at field frequencies where $\omega \tau \gg 1$. Finally, $\Delta \varepsilon_{n}=\varepsilon_{s}-\varepsilon_{\infty}$, where $\varepsilon_{s}$ is the permittivity of the tissue at field frequencies where $\omega \tau \ll 1$. These parameters are reported in [33] for a large variety of body tissues.

The implantation areas have been investigated by using the Visible Human Server [34], that enables 3D real time navigation into the human body. Four different locations, such as arm, forearm, abdomen, and leg have been explored with the Visible Human Server and modeled using the Cole-Cole dispersion model [32]. The description of the tissues within these implantation areas is reported in Table I, together with the location of the coils. The different tissues have been imported in the simulation tool Agilent Momentum to perform full-wave analyses in different parts of the human body. The power links have been considered as 2-port networks and the S-parameters obtained with the simulation tool have been used to calculate the link efficiency. The results of those simulations are presented in the following subsections.

\section{B. Working Frequency}

Most of the inductive links presented in the literature operate at frequencies of few megahertz [10]-[15], [21], [22], [35], generally below $10 \mathrm{MHz}$. This upper limit is often adopted to minimize the quantity of power absorbed by the tissues and increase the link efficiency. The power absorbed per unit mass of tissue due to an incident electromagnetic field is defined as Specific Absorption Rate (SAR). Different studies have shown that the whole-body averaged SAR in an average man is minimum for frequencies below $10 \mathrm{MHz}$, reaches the maximum around $70 \mathrm{MHz}$ and decreases for microwave frequencies [36], [37].

The optimal frequency for an inductive link is closely related to the geometry of the inductors. Indeed, a reduction of the inductor size shifts the optimal frequency towards higher values [38]. However, the drawback of this choice is a considerably 
TABLE I

IMPLANTATION SITES [32]

\begin{tabular}{|c|c|c|c|}
\hline Arm & Forearm & Abdomen & Leg \\
\hline $\begin{array}{c}\text { External coil } \\
\text { Air-1 } \mathrm{mm} \\
\text { Skin- } 1.5 \mathrm{~mm} \\
\text { Fat }-4.5 \mathrm{~mm} \\
\text { Air-1 } \mathrm{mm} \\
\text { Receiving coil } \\
\text { Air-1 } \mathrm{mm} \\
\text { Fat- } 4.5 \mathrm{~mm} \\
\text { Muscle-36 mm } \\
\text { Humerus }-18 \mathrm{~mm} \\
\text { Muscle }-48 \mathrm{~mm} \\
\text { Fat }-9 \mathrm{~mm} \\
\text { Skin }-1.5 \mathrm{~mm}\end{array}$ & $\begin{array}{c}\text { External coil } \\
\text { Air-1 } \mathrm{mm} \\
\text { Skin- } 2 \mathrm{~mm} \\
\text { Fat-1.5 mm } \\
\text { Air-1 } \mathrm{mm} \\
\text { Receiving coil } \\
\text { Air-1 } \mathrm{mm} \\
\text { Fat }-1.5 \mathrm{~mm} \\
\text { Muscle-12 mm } \\
\text { Ulna-5 mm } \\
\text { Muscle-2 mm } \\
\text { Radius }-3 \mathrm{~mm} \\
\text { Muscle-12 mm } \\
\text { Blood }-5 \mathrm{~mm} \\
\text { Muscle- } 4 \mathrm{~mm} \\
\text { Blood }-4 \mathrm{~mm} \\
\text { Muscle- } 8 \mathrm{~mm} \\
\text { Fat }-3 \mathrm{~mm} \\
\text { Skin- } 2 \mathrm{~mm}\end{array}$ & $\begin{array}{c}\text { External coil } \\
\text { Air-1 mm } \\
\text { Skin-2 mm } \\
\text { Fat- } 2 \mathrm{~mm} \\
\text { Air-1 mm } \\
\text { Receiving coil } \\
\text { Air-1 } \mathrm{mm} \\
\text { Fat- } 2 \mathrm{~mm} \\
\text { Muscle-17 mm } \\
\text { Colon- } 40 \mathrm{~mm} \\
\text { Fat- } 40 \mathrm{~mm} \\
\text { Intestine-33 mm } \\
\text { Blood-17 mm } \\
\text { Spine- } 56 \mathrm{~mm} \\
\text { Muscle- } 83 \mathrm{~mm} \\
\text { Fat }-10 \mathrm{~mm} \\
\text { Skin- } 2 \mathrm{~mm}\end{array}$ & $\begin{array}{c}\text { External coil } \\
\text { Air-1 mm } \\
\text { Skin- } 2 \mathrm{~mm} \\
\text { Fat-3 mm } \\
\text { Air-1 mm } \\
\text { Receiving coil } \\
\text { Air-1 mm } \\
\text { Fat-3 mm } \\
\text { Muscle-45 mm } \\
\text { Femur-14 mm } \\
\text { Muscle- } 45 \mathrm{~mm} \\
\text { Fat- } 6 \mathrm{~mm} \\
\text { Skin- } 2 \mathrm{~mm}\end{array}$ \\
\hline
\end{tabular}
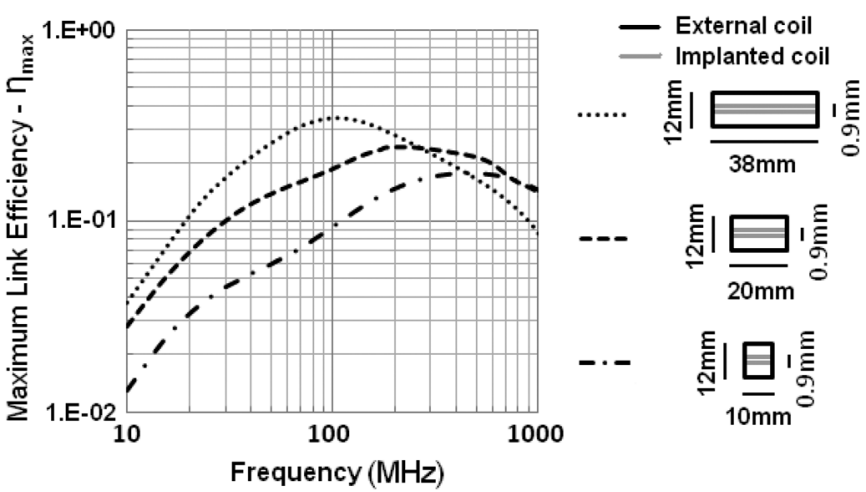

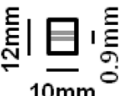

Frequency $(\mathrm{MHz})$

Fig. 3. The reduced size of the coils causes a higher optimal frequency, along with a reduced maximum link efficiency. Simulations are performed in the arm. Reprinted from [32].

smaller link efficiency, as compared to bigger geometries operating at lower frequencies, below $10 \mathrm{MHz}$.

This effect is simulated in Fig. 3, from [32], where the link efficiencies of three pairs of small-size, single-turn inductors are compared. The receiving coils are single-turn, rectangular structures with copper metal traces. The short side is set equal to $0.9 \mathrm{~mm}$; this value is compatible with the diameter of commercial needles. The trace has a thickness of $38 \mu \mathrm{m}$ and a width of $0.2 \mathrm{~mm}$. The external coil has copper traces with width equal to $2 \mathrm{~mm}$ and thickness equal to $38 \mu \mathrm{m}$. For all of the inductors, the optimum frequency is fairly higher than the range commonly used. Moreover, by reducing the size of the inductors, the optimum frequency increases and the maximum link efficiency decreases.

The effects of different implantation sites on the maximum link efficiency are shown in Fig. 4, from [32]. Different implantation sites involve different body tissues and implantation depths. However, these factors do not significantly affect the optimum frequency, that is mostly determined by the geometry of the inductors. The differences of value among the three curves are mostly due to the different implantation depths.

Finally, if a larger, multi-turn spiral inductor is used as transmitter, while maintaining fixed the size of the receiver, the op-

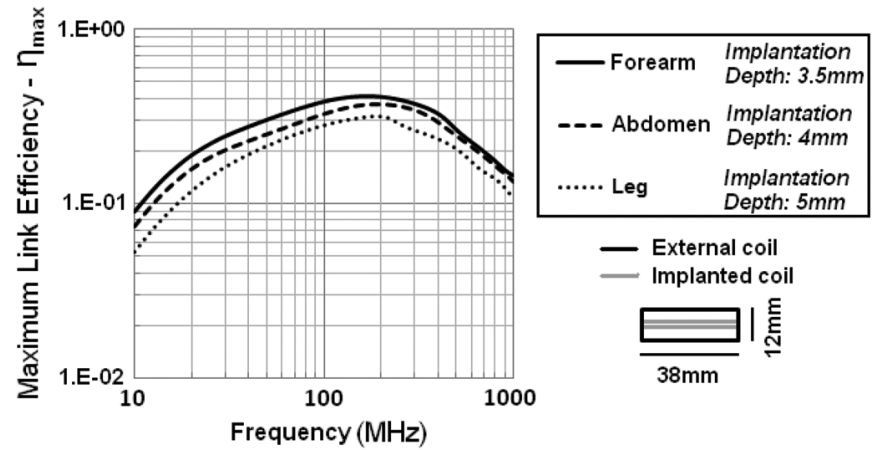

Fig. 4. Different implantation sites do not affect the optimum frequency. The differences among the three curves are mostly due to the different implantation depths. Reprinted from [32].

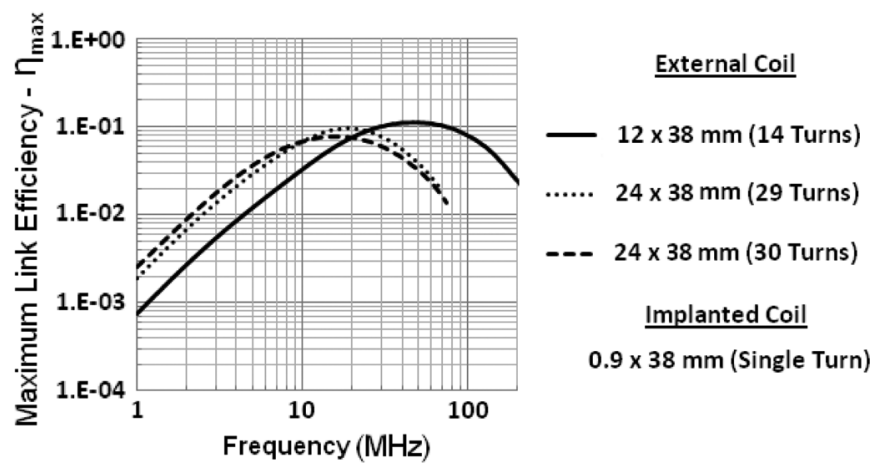

Fig. 5. Maximum link efficiency obtained with multi-turn external coils. The number of turns and the geometry of the external coils affect the optimal frequency. Simulations are performed in the arm. Reprinted from [32].

timal frequency shifts down in the range of frequencies where absorption is minimum (Fig. 5, from [32]). In Fig. 5, for two curves (14 turns and 29 turns), the trace width of the external coil and the spacing between adjacent traces is $0.2 \mathrm{~mm}$; in the third curve (30 turns) the trace width of the external coil is $0.25 \mathrm{~mm}$, while the spacing between adjacent traces is $0.14 \mathrm{~mm}$. This last simulation confirms the possibility to use a small receiving inductor, with the size and the shape of a needle, and to operate at frequencies of few megahertz if a multi-turn external inductor is used.

\section{Link Efficiency}

Different geometries have been designed and measured, to determine the optimal tradeoff among the link efficiency, the voltage gain, and the size of the receiving inductors. The curves presented in this subsection are obtained by measuring the electrical parameters of the coils. By substituting these parameters in (9) and (10), the link efficiency and the voltage gain are determined. The electrical parameters of the coils are measured by means of a network analyzer (Rohde \& Schwarz-ZVL).

Initially, receiving inductors with different size have been tested by using the same external inductor (Fig. 6). The results of these measurements show that the link efficiency does not increase over $10 \mathrm{MHz}$. This behavior is in agreement with the simulations in Fig. 5. Moreover, by increasing the size and the number of turns of the receiving inductor, both the link efficiency and the voltage gain increase. Indeed, a larger area and 

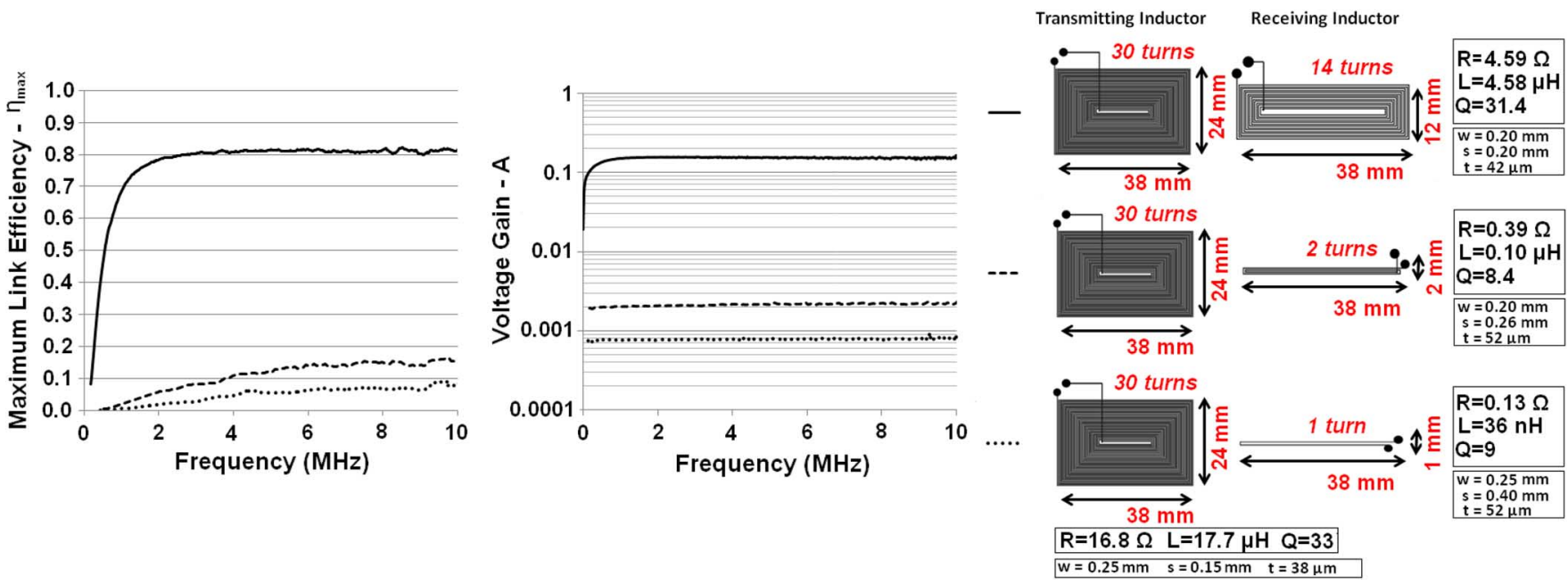

Fig. 6. Link efficiency and voltage gain decrease when smaller receiving inductors are used. The distance between the coils is $5 \mathrm{~mm}$. The inductance and resistance of each coil are measured at $5 \mathrm{MHz}$. Notations $w, s$, and $t$ indicate the trace width, the spacing between adjacent turns, and the trace thickness, respectively.
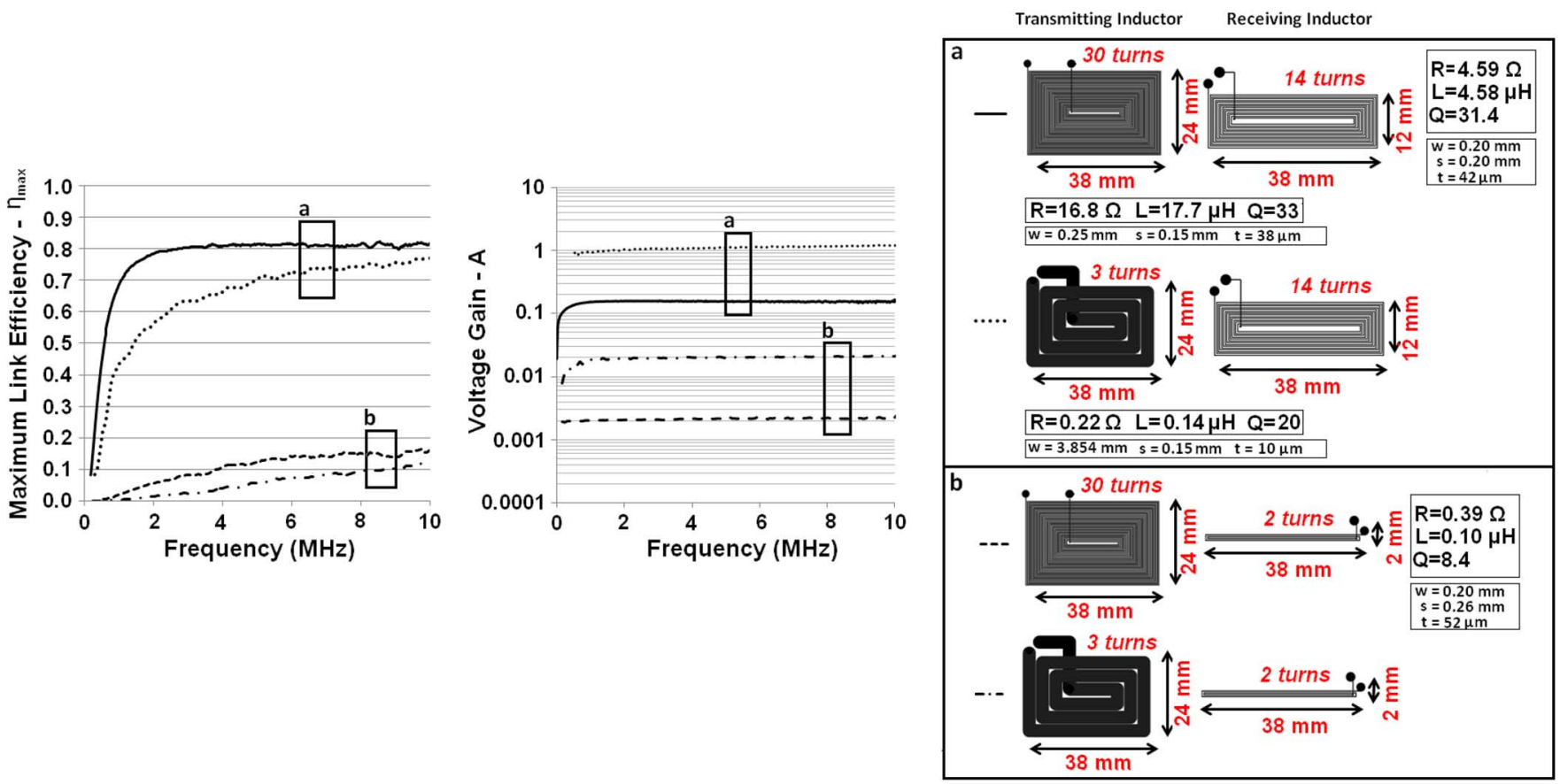

Fig. 7. By reducing the number of turns of the external inductor and increasing their width, the link efficiency slightly decreases while the voltage gain noticeably increases. This behavior is not affected by the larger (group a) or smaller (group b) size of the internal inductor. The distance between the coils is $5 \mathrm{~mm}$. The inductance and resistance of each coil are measured at $5 \mathrm{MHz}$. The notation of the parameters is the same as in Fig. 6.

more turns for the receiving inductor lead to a larger mutual inductance $M$ between the coils. The link efficiency (9) increases with $M$. Furthermore, if expression (7) is satisfied, also the voltage gain (10) increases with $M$.

Different widths of the traces have been measured for the external inductor, while maintaining the area fixed (Fig. 7). A lower number of turns with larger width results in a smaller resistance $R_{1}$ of the external inductor and the maximum link efficiency (9) is a decreasing function of $R_{1}$. However, a lower number of turns also reduces the mutual inductance $M$ and the maximum link efficiency (9) is an increasing function of $M$. Consequently, to test which behavior is predominant between the decrease in $R_{1}$ and the decrease in $M$, different widths are tested for the traces of the external inductor, while maintaining the inductor area fixed. In the inductors with wider traces, the decrease in $M$ outweighs the decrease in $R_{1}$, thereby lowering the maximum link efficiency. Instead, the voltage gain strongly increases where the number of turns of the external inductor is reduced. Indeed, it can be shown that the voltage gain (10) is inversely proportional to the number of turns of the external coil under a constant-Q assumption [17].

This assumption considers the quality factor of a coil independent of the number of turns, for a given shape and size of the coil. This assumption can be used while optimizing the link efficiency, since the maximum link efficiency varies slowly with the quality factors of the inductors composing the link. This 


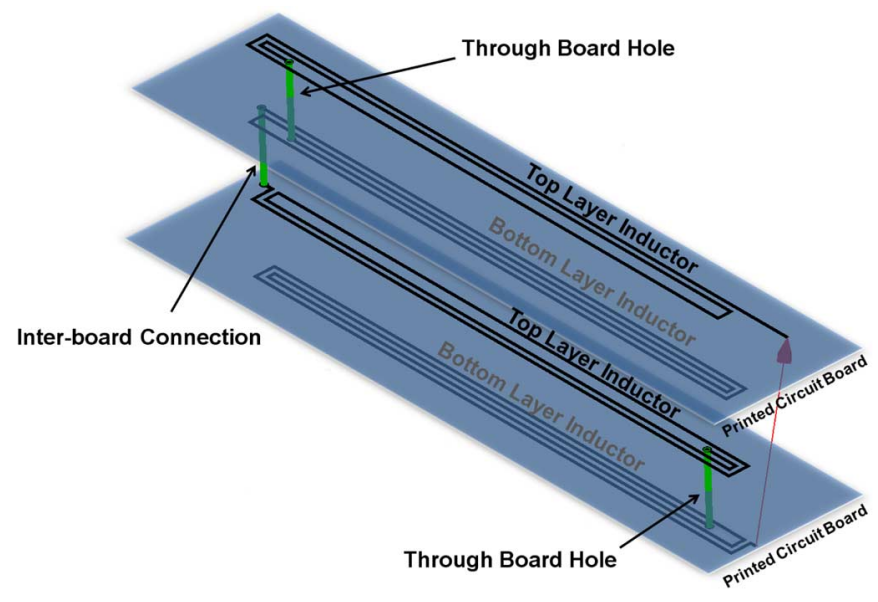

Fig. 8. Layout of a multi-layer spiral inductor on printed circuit board.

assumption has been used in [17], [39] to optimize the link performance.

\section{Multi-Layer Inductors}

The major constraint associated with the use of subcutaneous implantable devices is the size. Indeed, to reduce the discomfort of the patient and facilitate the implantation process, implantable devices should be as small as possible. This requirement is in conflict with the results shown in Fig. 6. Indeed, a smaller internal coil results in a lower link efficiency and voltage gain. To mitigate this issue, a multi-layer approach is experimentally investigated. Multi-layer inductors, thanks to their small area, are particularly suitable for this type of applications. Moreover, the use of rectangular multi-layer inductors facilitates their injection in the subcutaneous zones by means of a syringe.

The design of a multi-layer inductor is shown in Fig. 8. In a multi-layer coil, the same spiral inductor is replicated on the two layers of several printed circuit boards; then, the different boards are stacked and the inductors are electrically connected. Thus, a multi-layer inductor is obtained. A multi-layer inductor has the same area as the equivalent single layer inductor, but includes many more turns and, hence, a higher coupling with the external inductor. Attention must be paid to the current direction among the different layers. Indeed, layout must be designed to have the current flowing in the same direction in all the layers. Thus, the contribution of each layer to the total magnetic field will have the same sign.

Several multi-layer inductors are realized and tested to investigate their performance and to compare them with a singlelayer approach (Fig. 9). In Fig. 10 the same geometries depicted in Fig. 6 are shown, with the addition of an 8-layer, 14-turn inductor. This multi-layer inductor has higher link efficiency and voltage gain as compared with the single-layer inductor having the same area. The link efficiency is $35 \%$ higher, shifting from 0.17 to 0.23 at $10 \mathrm{MHz}$. The voltage gain is almost one order of magnitude higher.

By using multi-layer inductors it is possible to partially compensate a reduction of area by properly increasing the number of layers. This possibility is shown in Fig. 10. The 12-layer, 21-turn inductor having an area of $30 \mathrm{~mm}^{2}$ and a total thick-

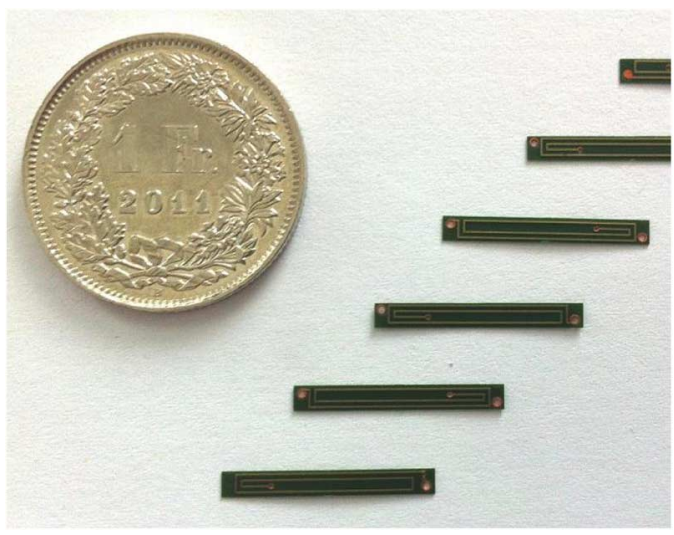

Fig. 9. Multi-layer spiral inductors are obtained by stacking and connecting single-layer inductors realized on a flexible substrate.

ness of $816 \mu \mathrm{m}$, exhibits almost the same performance of the 8-layer, 14-turn inductor, with an area of $76 \mathrm{~mm}^{2}$ and a total thickness of $544 \mu \mathrm{m}$. Consequently, the same performance is obtained with an area reduction of about $60 \%$.

\section{EXPERIMENTAL RESULTS}

A system is designed to power the implantable sensor and provide bidirectional communication [16]. The system is realized with commercial off-the-shelf components on a flexible substrate, in order to be embedded into a skin patch and placed directly over the implantation area. The resulting device has been shown in Fig. 1.

In the next subsections the schematic of the system is depicted, along with the description of an implantable target device. The performance of receiving multi-layer spiral inductors is measured. Finally, a comparison with the results presented in literature is given.

\section{A. Schematic Description}

As previously reported, the system has been conceived to be embedded into a skin patch, in order to be easily placed over the implantation area [16]. Thin lithium-ion polymer batteries power the system, that can be remotely controlled through a bluetooth module. The mains blocks of that device are summarized in Fig. 11. In this section, a high level description of the system is reported. Further implementation details can be found in [16].

Power is transmitted through the inductive link by using a class-E power amplifier. Amplifiers working in class-E are preferred to drive inductive links, due to their high efficiency, theoretically equal to $100 \%$. The schematic of the class-E power amplifier is shown in Fig. 12. The MOSFET $M_{1}$, driven by a square waveform, acts as a switch. If capacitors $C_{S}$ and $C_{P}$ are properly tuned, the current $i_{s}$ through the switch and the voltage $v_{s}$ across the switch are never different from zero at the same time. Therefore, theoretically the switch does not dissipate power. An analytical description of class-E amplifiers can be found in [17], [40].

The system is conceived to power implantable biosensors dedicated to the lactate monitoring. An example of an implantable device is given in [41]. That device is designed to 

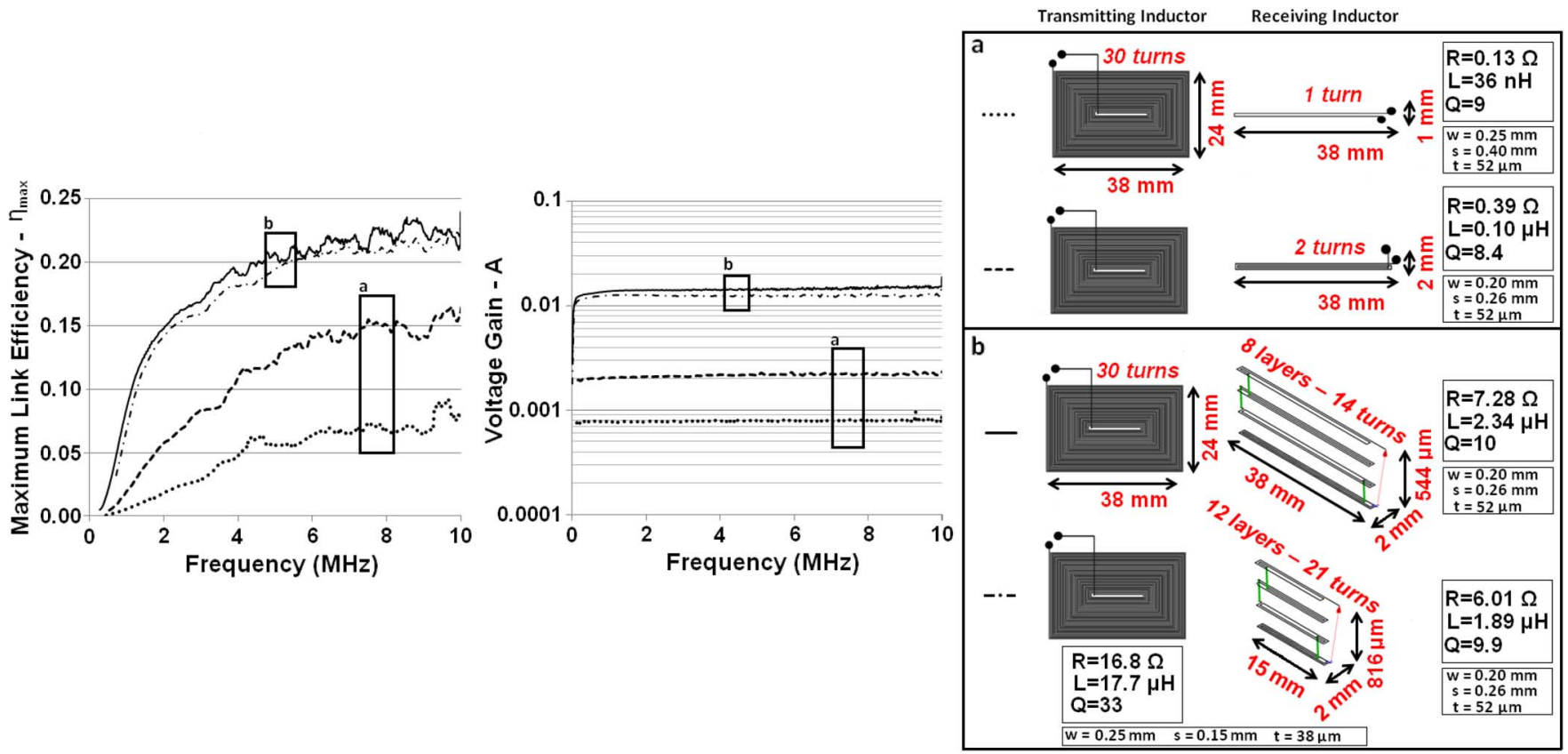

Fig. 10. Multi-layer spiral inductors (group b) enable a higher link efficiency and voltage gain if compared with single-layer inductors having the same area (group a). Furthermore, the reduction of area in multi-layer inductors can be partially compensated by increasing the number of layers (group b). The distance between the coils is $5 \mathrm{~mm}$. The inductance and resistance of each coil are measured at $5 \mathrm{MHz}$. The notation of the parameters is the same as in Fig. 6 .

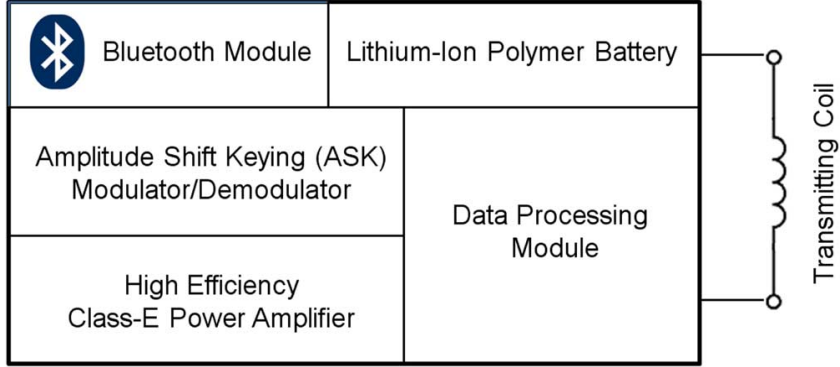

Fig. 11. Schematic illustration of the system to remotely power an implanted device. Bidirectional data communication with the implanted device is achieved with ASK modulator/demodulator. Long-range communication is performed by using the bluetooth protocol. The system has been conceived to be embedded into a skin patch.

detect endogenous compounds, such as the lactate, by means of chronoamperometry. In this technique, a fixed potential, equal to the reduction or oxidation potential of the lactate, is applied to the solution; the current read by the sensor is proportional to the lactate concentration. The proposed circuit has been realized in $0.18 \mu \mathrm{m}$ CMOS technology. The supply voltage is $1.8 \mathrm{~V}$ and its maximum current is about $300 \mu \mathrm{A}$, leading to a power consumption of $540 \mu \mathrm{W}$.

To enhance the versatility of the transmitter and to be able to power different families of implantable devices the maximum transmitted power is fixed at $10 \mathrm{~mW}$. This value is used with the design expressions in [17], [40] to determine the appropriate $V_{C C}, C_{S}$, and $C_{P}$ (Fig. 12). The working frequency of the transmitter is fixed at $5 \mathrm{MHz}$.

The amplitude modulator to perform downlink communication, also shown in Fig. 12, is based on that in [42]. This circuit, based on a Darlington pair, modulates the power carrier

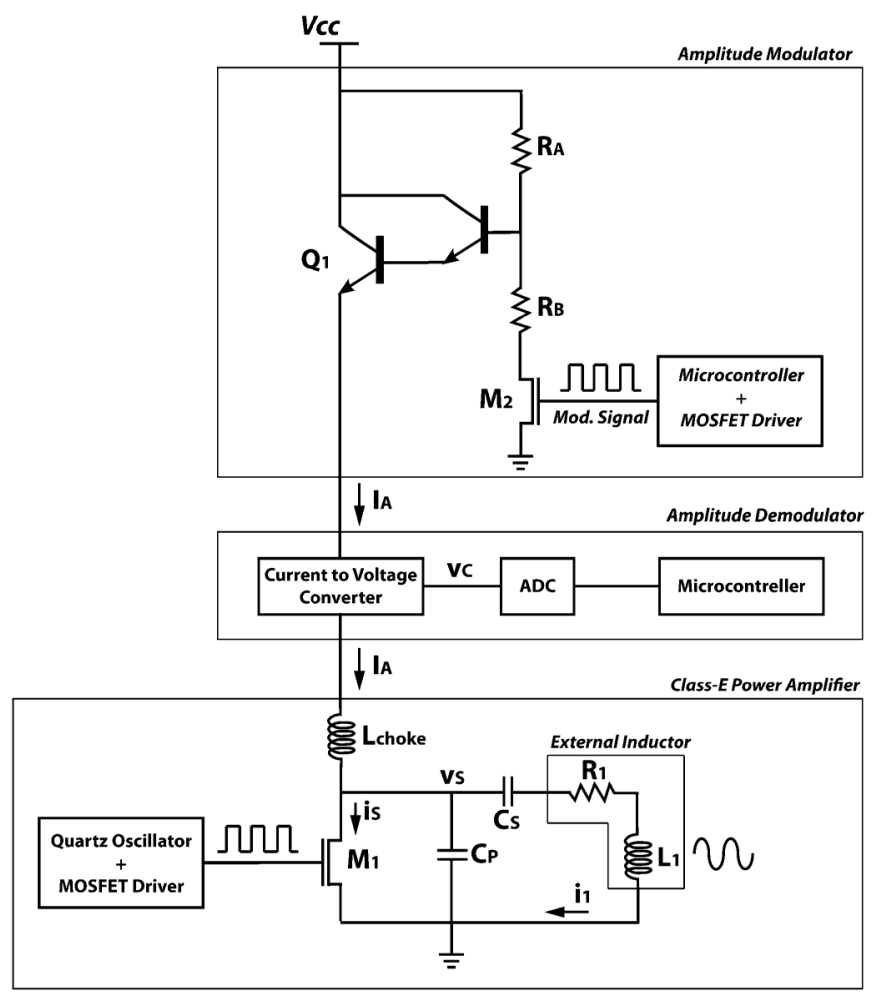

Fig. 12. Schematic of the external system. Class-E power amplifier, amplitude modulator and demodulator are shown.

across the external inductor by acting on the supply voltage of the class-E amplifier. The modulation depth is determined by the ratio between resistors $R_{A}$ and $R_{B}$. The modulation of the power carrier is detected by an amplitude demodulator embedded into the implantable sensor. 
Downlink communication is used to transmit commands, such as duration of the measurements and sample-rate, to the implantable sensor. By coding a single instruction as a byte, a downlink bit-rate of few kilobytes per second enables a setup time of the sensor in the order of few milliseconds. Thus, the desired downlink bit-rate is set to $10 \mathrm{kbps}$.

Uplink communication is obtained by changing the internal load of the receiving coil. This change affects the load seen by the external coil, thereby varying the current flowing on it. Thus, by modulating the internal load, uplink communication is performed without any implantable RF transmitter. This technique is often named backscattering. The load modulation affects not only the current flowing on the external inductor, but also the DC current $I_{A}$ drawn by the amplifier from the supply. To demodulate an LSK bitstream coming from uplink communication, current $I_{A}$ is converted into a voltage, digitized and analyzed by a microcontroller (Fig. 12). High current $I_{A}$ corresponds to an internal load not short-circuited (digital value 0 ); low $I_{A}$ corresponds to an internal load short-circuited (digital value 1). Since the value of $I_{A}$ strongly depends on several factors, such as the distance between the inductors and the interleaved tissues, the threshold between high and low current is updated prior to each communication.

Lactate concentration in body fluids exhibits a weak temporal dependence. A measurement rate of one sample per minute is sufficient to track with precision the lactate concentration of an athlete under stress. Thus, an uplink bit-rate of few bytes per minute would be sufficient. However, in order to communicate efficiently with the external unit during the setup time, the uplink bit-rate of the sensor is set to $10 \mathrm{kbps}$, equal to the downlink bit-rate.

Digital circuitry, based on a microcontroller, drives the class-E power amplifier and generates the modulating signal. Furthermore, it monitors the current $I_{A}$. The microcontroller is remotely driven by means of a bluetooth module and can bidirectionally communicate with a remote device.

The entire system is powered with two rechargeable thin lithium-ion polymer batteries [43]. On board, different voltages are generated for the analog and the digital circuits.

\section{B. Setup}

In order to measure the performance of the system with multi-layer spiral inductors, the following setup is used. The transmitting inductor is driven by the class-E power amplifier previously presented. The power received by the internal inductor is measured by means of a spectrum analyzer (Rohde \& Schwarz-ZVL). The receiving inductor is not directly linked to the spectrum analyzer. Instead, a capacitive matching network is used to match the input impedance of the instrument $(50 \Omega)$ to the impedance of the receiving inductor.

A mechanical setup is used to adjust the distance between the transmitting and receiving inductors, while assuring a planar alignment. The distance between the inductors lies within the range from $6 \mathrm{~mm}$ to $70 \mathrm{~mm}$.

Downlink communication is tested by transmitting an ASK bitstream with the external patch and by reading it on the amplitude demodulator embedded into the spectrum analyzer.

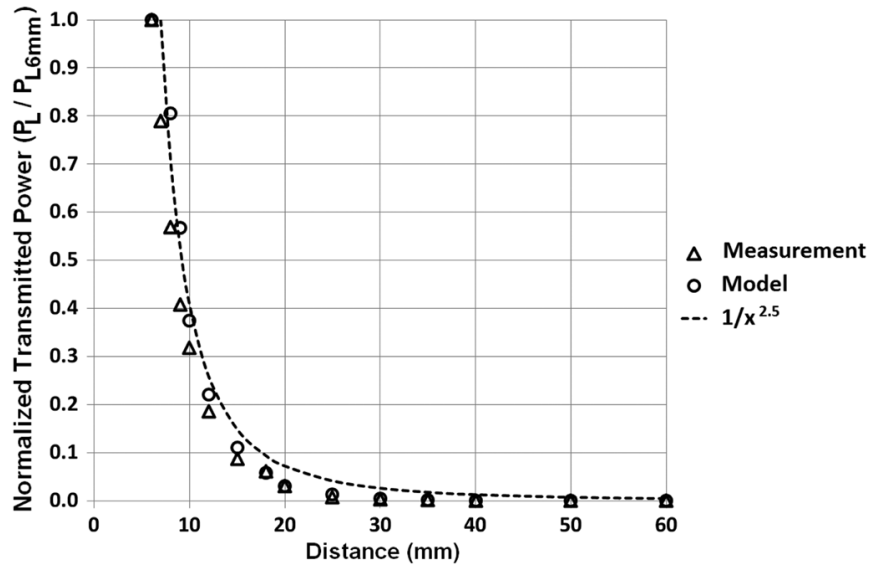

Fig. 13. Both the model based on (9) and the measurements confirm a strong dependency of the transferred power with distance.

Finally, uplink communication is tested by short-circuiting the $50 \Omega$ input of the spectrum analyzer according to a given bitstream and by reading that bitstream at the output of the current-to-voltage converter embedded into the patch (Fig. 12).

\section{Measurements}

To investigate the performance of the system, a first set of measurements is performed in ambient air. The receiving inductor is the 12-layer, 21-turn, $15 \times 2 \mathrm{~mm}^{2}$ coil shown in Fig. 10 .

The amplifier can transmit up to $15 \mathrm{~mW}$ over a distance of $6 \mathrm{~mm}$. The link efficiency, as calculated from (9) by using the measured values of the inductors, is $13 \%$. While transmitting $15 \mathrm{~mW}$ at $6 \mathrm{~mm}$, the amplifier consumes $340 \mathrm{~mW}$. Thus, the overall efficiency of the system is $4.4 \%$.

The transferred power quickly decreases when the distance between the inductors increases. In Fig. 13, the curve denoted as "model" is obtained by using the measured values of the inductors in (9); the curve named "measurement" is obtained by reporting the power effectively delivered to the load. Measurements present good agreement with the model reported in Section II. Both the curves decrease as $1 / x^{2.5}$.

A second set of measurements is performed using beef sirloin between the inductors (Fig. 14). The thickness of the sirloin is $17 \mathrm{~mm}$. In this case, the transferred power is $1.17 \mathrm{~mW}$. This result is similar to that obtained in ambient air by using the same distance between the inductors (Fig. 13).

Finally, data communication is investigated. Downlink communication is successful up to $100 \mathrm{kbps}$. This rate is one order of magnitude higher than the desired one, fixed at $10 \mathrm{kbps}$. Higher bit-rates are still possible, but not useful for the target application (detection of lactate). The same bit-rate (100 kbps) is obtained by using beef sirloin between the inductors. In Fig. 15(a) is shown a downlink bitstream, having a bit-rate of $100 \mathrm{kbps}$, detected on the receiving inductor. The bitstream is recorded at the output of the amplitude demodulator.

Uplink communication is performed at $66.6 \mathrm{kbps}$ up to $1.1 \mathrm{~cm}$ of distance between the coils. The uplink bit-rate is limited by the computational time needed by the microcontroller to distinguish the two logic levels, according to the threshold value measured before the communication. Similarly to the downlink 
TABLE II

Comparison Among Inductive Powering Systems Dedicated to ImPlantable Devices

\begin{tabular}{|c|c|c|c|c|c|c|c|}
\hline Ref. & Coil Area & Coil Thickness & $\begin{array}{c}\text { Carrier } \\
\text { Frequency }\end{array}$ & $\begin{array}{c}\text { Data } \\
\text { Transmission }\end{array}$ & $\begin{array}{c}\text { Transmitted } \\
\text { Power }\end{array}$ & $\begin{array}{c}\text { Link } \\
\text { Efficiency }\end{array}$ & Distance \\
\hline [11] & $\begin{array}{l}\text { Tx: } 1960 \mathrm{~mm}^{2} \\
\mathrm{Rx}: 314 \mathrm{~mm}^{2}\end{array}$ & $\begin{array}{l}\text { Tx: } 9.5 \mathrm{~mm} \\
\mathrm{Rx}: 5 \mathrm{~mm}\end{array}$ & $4 \mathrm{MHz}$ & Uplink & $11 \mathrm{~mW}$ & & $28 \mathrm{~mm}$ \\
\hline [12] & $\begin{array}{l}\text { Tx: } 1.32 \cdot 10^{5} \mathrm{~mm}^{2} \\
\text { Rx: } 79 \mathrm{~mm}^{2}\end{array}$ & $\begin{array}{l}\text { Tx: } 300 \mathrm{~mm} \\
\mathrm{Rx}: 13 \mathrm{~mm}\end{array}$ & $1 \mathrm{MHz}$ & & $150 \mathrm{~mW}$ & $1 \%$ (min.) & $205 \mathrm{~mm}$ \\
\hline [13] & $\begin{array}{l}\text { Tx: } 1963 \mathrm{~mm}^{2} \\
\text { Rx: } 35 \mathrm{~mm}^{2}\end{array}$ & $\begin{array}{c}\mathrm{Tx}: 5 \mathrm{~mm} \\
\mathrm{Rx}: 1.9 \mathrm{~mm}\end{array}$ & $5 \mathrm{MHz}$ & Downlink & $10 \mathrm{~mW}$ & & $40 \mathrm{~mm}$ \\
\hline [44] & $\begin{array}{c}\text { Tx: } 400 \mathrm{~mm}^{2} \\
\mathrm{Rx}: 4 \mathrm{~mm}^{2}\end{array}$ & $\begin{array}{l}\text { Tx: } 3.8 \cdot 10^{-2} \mathrm{~mm} \\
\mathrm{Rx}: 3.8 \cdot 10^{-2} \mathrm{~mm}\end{array}$ & $915 \mathrm{MHz}$ & & $0.14 \mathrm{~mW}$ & $0.06 \%$ (max.) & $15 \mathrm{~mm}$ \\
\hline $\begin{array}{c}\text { Present } \\
\text { work }\end{array}$ & 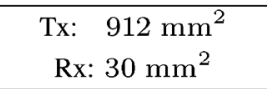 & $\begin{array}{l}\text { Tx: } 0.136 \mathrm{~mm} \\
\mathrm{Rx}: 0.816 \mathrm{~mm}\end{array}$ & $5 \mathrm{MHz}$ & Bidirectional & $15 \mathrm{~mW}$ & $4.4 \%(\max )$ & $6 \mathrm{~mm}$ \\
\hline
\end{tabular}

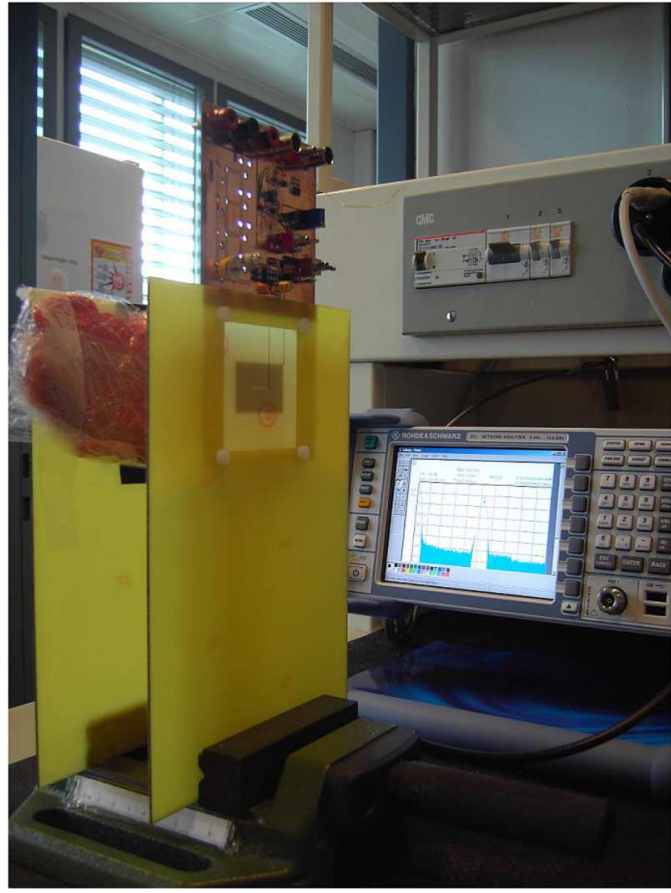

Fig. 14. Measurements are performed using beef sirloin between the inductors. Reprinted from [16].

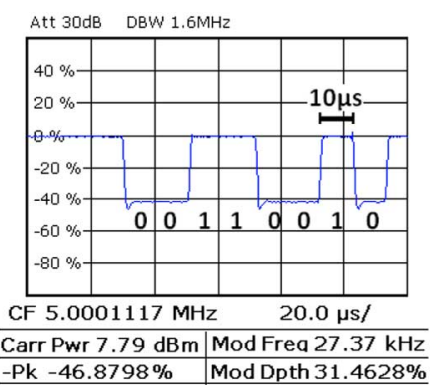

(a)

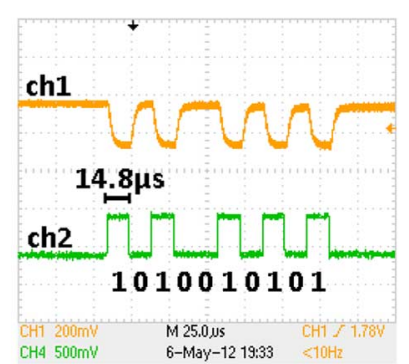

(b)
Fig. 15. A downlink bitstream, having a bit-rate of $100 \mathrm{kbps}$, is detected by the receiving inductor (a). An uplink bitstream, having a bit-rate of $66.6 \mathrm{kbps}$, is recorded at the output of the current-to-voltage converter (channel 1) while a modulating signal (channel 2) short-circuits the internal inductor (b).

communication, the bit-rate achieved in the uplink communication is almost one order of magnitude higher than the desired one, fixed at $10 \mathrm{kbps}$. In Fig. 15(b) is shown an uplink bitstream, having a bit-rate of $66.6 \mathrm{kbps}$, received by the external system. The uplink bitstream (channel 1) is recorded with an oscilloscope at the output of the current-to-voltage converter of Fig. 12. The signal that modulates the internal load is also reported (channel 2).

Data transmission affects the power transfer. While downlink communication is not performed, the amplifier is directly connected to $V_{C C}$, thus bypassing the amplitude modulator. While a high logic level is transmitted, the use of the amplitude modulator decreases the received power of about $33 \%$ when compared to the case where the amplifier is directly connected to $V_{C C}$. When a low logic level is transmitted, the received power decreases of about $82 \%$ due to the reduced supply voltage. During uplink communication, load modulation detunes the amplifier, thus reducing the link efficiency.

However, in this specific application the communication between the sensor and the system is sporadic. Downlink communication is performed for the initial setup of the sensor; uplink communication is performed only few milliseconds every minute. Thus, the effects of data communication on power transfer are negligible.

\section{Comparison With the State of the Art}

The system realized is compared in Table II with similar devices presented in the literature [11]-[13], [44]. The use of multi-layer spiral inductors, described in the previous section, enables a higher link efficiency and transmitted power, if compared with single-layer spiral inductors of the same area. This characteristic, combined with the low thickness, makes multi-layer spiral inductors particularly suitable for low invasive implanted devices.

\section{CONCLUSIONS}

The use of multi-layer printed spiral inductors to remotely power implantable sensors is investigated. Multi-layer structures are implemented on flexible substrates to facilitate the implantation process. Furthermore, it is possible to partially compensate a reduction of the inductor area by properly increasing the number of layers. Differently from the planar microfabricated inductors, no technological limitations exist for the number of layers that can be stacked. Multi-layer, printed 
spiral inductors with rectangular shape have been studied and measured. The rectangular shape, having the form factor of a needle, is designed to facilitate the implantation process and reduce the discomfort of the patient.

A system conceived to be embedded into a skin patch is designed to verify the performance of those inductors. A high efficiency class-E power amplifier is utilized to drive the external inductor. Powered by two thin lithium-ion polymer batteries, the system can transfer up to $15 \mathrm{~mW}$ over a distance of $6 \mathrm{~mm}$ in air. The maximum link efficiency measured is $4.4 \%$. Furthermore, the system can transfer up to $1.17 \mathrm{~mW}$ when a $17 \mathrm{~mm}$ beef sirloin is placed between the inductors. Bidirectional data communication between the system and the implantable device is performed. Downlink communication is achieved by means of an external amplitude modulator. Downlink bit-rate up to $100 \mathrm{kbps}$ is demonstrated. Uplink communication is based on the backscattering technique and is performed at $66.6 \mathrm{kbps}$ up to a distance of $1.1 \mathrm{~cm}$ between the inductors.

These results are obtained by using multi-layer spiral inductors as receivers. If compared with single-layer inductors having the same area, multi-layer inductors exhibit a link efficiency improvement up to $35 \%$ and a voltage gain improvement of one order of magnitude. The overhead for these improvements is an increase in thickness by a few hundreds micrometers. Moreover, the same performance, in terms of link efficiency and voltage gain, is obtained with decrease in area of $60 \%$ by simply increasing the number of layers.

Finally, the system is compared with the state of the art. Due to multi-layer inductors, the volumes of the coils can be scaled down without reducing the link efficiency and the transmitted power.

\section{ACKNOWLEDGMENT}

J. Olivo thanks Dr. F. Merli for the useful discussions on multi-layer inductors and the kind suggestions and Dr. V. Pavlidis for the assistance in preparing the article.

\section{REFERENCES}

[1] A. Poscia, D. Messeri, D. Moscone, F. Ricci, and F. Valgimigli, "A novel continuous subcutaneous lactate monitoring system," Biosens. Bioelectron., vol. 20, pp. 2244-2250, 2005.

[2] M. Romero, F. Garay, and A. Baruzzi, "Design and optimization of a lactate amperometric biosensor based on lactate oxidase cross-linked with polymeric matrixes," Sensors Actuat. B-Chem., vol. 131, pp. 590-595, 2008.

[3] A. Guiseppi-Elie, S. Brahim, G. Slaughter, and K. Ward, "Design of a subcutaneous implantable biochip for monitoring of glucose and lactate," IEEE Sensors J., vol. 5, no. 3, pp. 345-355, 2005.

[4] J. Huang, Z. Song, J. Li, Y. Yang, H. Shi, B. Wu, J. Anzai, T. Osa, and Q. Chen, "A highly-sensitive L-lactate biosensor based on sol-gel film combined with Multi-walled Carbon Nanotubes (MWCNTs) modified electrode," Mater. Sci. Eng. C, vol. 27, pp. 29-34, 2007.

[5] X. Cui, C. Li, J. Zang, and S. Yu, "Highly sensitive lactate biosensor by engineering Chitosan/pvi-os/cnt/lod network nanocomposite," Biosens. Bioelectron., vol. 22, pp. 3288-3292, 2007.

[6] C. Lam, J. James, R. McCluskey, and R. Hunter, "Pulmonary toxicity of single-wall carbon nanotubes in mice 7 and 90 days after intratracheal instillation," Toxicol. Sci., vol. 77, pp. 126-134, 2004.

[7] D. Warheit, B. R. Laurence, K. Reed, D. Roach, G. Reynolds, and T. Webb, "Comparative pulmonary toxicity assessment of single-wall carbon nanotubes in rats," Toxicol. Sci., vol. 77, pp. 117-125, 2004.
[8] J. Olivo, S. Carrara, and G. De Micheli, "Energy harvesting and remote powering for implantable biosensors," IEEE Sensors J., vol. 11, no. 7, pp. 1573-1586, 2010.

[9] Activa RC Recharging System 37751, Medtronic.

[10] T. Akin, K. Najafi, and R. Bradley, "A wireless implantable multichannel digital neural recording system for a micromachined sieve electrode," IEEE J. Solid-State Circuits, vol. 33, pp. 109-118, 1998.

[11] C. Sauer, M. Stanaćević, G. Cauwenberghs, and N. Thakor, "Power harvesting and telemetry in CMOS for implanted devices," IEEE Trans. Circuits Syst. I, Reg. Papers, vol. 52, pp. 2605-2613, 2005.

[12] B. Lenaerts and R. Puers, "An inductive power link for a wireless endoscope," Biosens. Bioelectron., vol. 22, pp. 1390-1395, 2007.

[13] G. Gudnason, E. Bruun, and M. Haugland, "A chip for an implantable neural stimulator," Analog Integr. Circuits Signal Process., vol. 22, pp. 81-89, 2000.

[14] B. Smith, Z. Tang, M. Johnson, S. Pourmehdi, M. Gazdik, J. Buckett, and P. Peckham, "An externally powered, multichannel, implantable stimulator-telemeter for control of paralyzed muscle," IEEE Trans. Biomed. Eng., vol. 45, pp. 463-475, 1998.

[15] M. Ahmadi and G. Jullien, "A wireless-implantable microsystem for continuous blood glucose monitoring," IEEE Trans. Biomed. Circuits Syst., vol. 3, pp. 169-180, 2009.

[16] J. Olivo, S. Carrara, and G. De Micheli, "IronIC patch: A wearable device for the remote powering and connectivity of implantable systems," in Proc. IEEE I2MTC Conf., 2012, pp. 286-289.

[17] B. Lenaerts and R. Puers, Omnidirectional Inductive Powering for Biomedical Implants. New York: Springer, 2009.

[18] G. Kendir, W. Liu, G. Wang, M. Sivaprakasam, R. Bashirullah, M. Humayun, and J. Weiland, "An optimal design methodology for inductive power link with class-E amplifier," IEEE Trans. Circuits Syst. I, Reg. Papers, vol. 52, pp. 857-866, 2005.

[19] C. Sullivan, "Optimal choice for number of strands in a Litz-wire transformer winding," IEEE Trans. Power Electron., vol. 14, pp. 283-291, 1999.

[20] A. Lotfi and F. Lee, "A high frequency model for litz wire for switchmode magnetics," in Proc IEEE IAS Conf. Rec., 1993.

[21] K. Silay, D. Dondi, L. Larcher, M. Declercq, L. Benini, Y. Leblebici, and C. Dehollain, "Load optimization of an inductive power link for remote powering of biomedical implants," in Proc. IEEE ISCAS Conf., 2009, pp. 533-536.

[22] J. Parramon, P. Doguet, D. Marin, M. Verleyssen, R. Muñoz, L. Leija, and E. Valderrama, "ASIC-based batteryless implantable telemetry microsystem for recording purposes," Proc. IEEE Int. Conf. IEEE Engineering in Medicine and Biology Society., vol. 5, pp. 2225-2228, 1997.

[23] A. Makharia and G. Rincón-Mora, "Integrating power inductors onto the IC-SOC implementation of inductor multipliers for DC-DC converters," in Proc. IEEE-IECON Conf. Rec., 2003, vol. 1, pp. 556-561.

[24] R. Merrill, T. Lee, H. You, R. Rasmussen, and L. Moberly, "Optimization of high Q integrated inductors for multi-level metal CMOS," in Proc. IEEE Int. Electron Devices Meeting Conf., 1995, pp. 983-986.

[25] C. Tang, C. Wu, and S. Liu, "Miniature 3-D inductors in standard CMOS process," IEEE J. Solid-State Circuits, vol. 37, pp. 471-480, 2002.

[26] Y. Choi and J. Yoon, "Experimental analysis of the effect of metal thickness on the quality factor in integrated spiral inductors for RF ICs," IEEE Electron Device. Lett., vol. 25, pp. 76-79, 2004

[27] J. Wu, V. Quinn, and G. Bernstein, "Powering efficiency of inductive links with inlaid electroplated microcoils," J. Micromech. Microeng., vol. 14, pp. 576-586, 2004.

[28] P. Basset, A. Kaiser, B. Legrand, D. Collard, and L. Buchaillot, "Complete system for wireless powering and remote control of electrostatic actuators by inductive coupling," IEEE/ASME Trans. Mechatronics, vol. 12, pp. 23-31, 2007.

[29] A. Laskovski, M. Yuce, and T. Dissanayake, "Stacked spiral for biosensor telemetry," IEEE Sensors J., vol. 11, pp. 1484-1490, 2011.

[30] A. Islam and S. Islam, "Design of multi-spiral solenoidal inductor for inductive power transfer in biomedical applications," in Proc. Applied Computational Electromagnetics Society Conf., 2011, pp. 369-374.

[31] J. Zhao, "A new calculation for designing multilayer planar spiral inductors," Electron. Design, Strategy, News, vol. 3, pp. 37-40, 2010.

[32] J. Olivo, S. Carrara, and G. De Micheli, "Optimal frequencies for inductive powering of fully implantable biosensors for chronic and elderly patients," in Proc. IEEE Sensors Conf., 2010, pp. 99-103. 
[33] S. Gabriel, R. Lau, and C. Gabriel, "The dielectric properties of biological tissues: III. Parametric models for the dielectric spectrum of tissues," Phys. Med. Biol., vol. 41, pp. 2271-2293, 1996.

[34] [Online]. Available: http://visiblehuman.epfl.ch

[35] M. Catrysse, B. Hermans, and R. Puers, "An inductive power system with integrated Bi-directional data-transmission," Sensors Actuat. A-Phys, vol. 115, pp. 221-229, 2004.

[36] S. Michaelson, "Health implications of exposure to radiofrequency/ microwave energies," Brit. J. Ind. Med, vol. 39, pp. 105-119, 1982.

[37] P. Dimbylow, "FDTD calculations of the whole-body averaged sar in an anatomically realistic voxel model of the human body from $1 \mathrm{MHz}$ to $1 \mathrm{GHz}$," Phys. Med. Biol., vol. 42, pp. 479-490, 1997.

[38] A. Poon, S. O'driscoll, and T. Meng, "Optimal frequency for wireless power transmission into dispersive tissue," IEEE Trans. Antennas Propag., vol. 58, pp. 1739-1750, 2010.

[39] W. Ko, S. Liang, and C. Fung, "Design of radio-frequency powered coils for implant instruments," Med. Biol. Eng. Comput., vol. 15, pp. 634-640, 1977.

[40] N. Sokal, "Class-E RF power amplifiers," $Q E X$, pp. 9-20, Jan./Feb. 2001.

[41] S. Ghoreishizadeh, S. Carrara, and G. De Micheli, "Circuit design for human metabolites biochip," in Proc. IEEE BioCAS Conf., 2011, pp. 460-463.

[42] B. Ziaie, S. Rose, M. Nardin, and K. Najafi, "A self-oscillating detuning-insensitive class-e transmitter for implantable microsystems," IEEE Trans. Biomed. Eng., vol. 48, pp. 397-400, 2001.

[43] H. Batteries, hp-lg325-0120-1s [Online]. Available: http://www.hyperion-eu.com

[44] S. O'Driscoll, A. Poon, and T. Meng, "A mm-sized implantable power receiver with adaptative link compensation," in Proc. ISSCC-Dig. Tech.Papers, 2009, pp. 294-295, 295a.

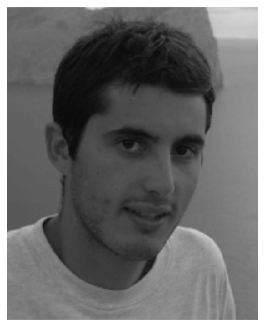

Jacopo Olivo received the M.Sc. degree in electrical engineering from the University of Bologna, Bologna, Italy, in 2008 and is currently working toward the Ph.D. degree at the Integrated System Laboratory, École Polytechnique Fédérale de Lausanne, Lausanne, Switzerland.

During his master project, he became involved in the field of biosensors concerning the design and development of an integrable system for electrochemical measurements in point-of-care applications for personalized medicine. His research interest focuses on the remote powering of implantable devices through inductive link.

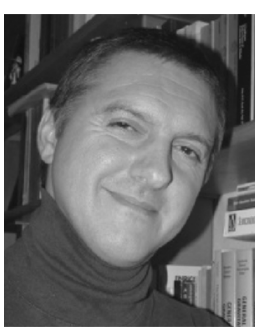

Sandro Carrara (M'09) received the M.Sc. degree from University of Genoa, Italy, in 1993, and the Ph.D. degree in biochemistry and biophysics from University of Padoa, Italy, in 1997.

$\mathrm{He}$ is Senior Scientist and Lecturer at the École Polytechnique Fédérale de Lausanne (EPFL), Lausanne, Switzerland, and Professor of nanobiosensing and micro/nano interfaces at the Department of Electrical Engineering and Biophysics (DIBE), University of Genoa, Italy. He has authored more than 100 publications and 10 patents. He authored Top 25 Hottest Articles (in 2004, 2005, 2008, and 2009) in highly-ranked international journals. His main scientific interest is in electrical phenomena mediated by nanostructured molecular thin films and the development of protein and DNA-based CMOS biochips.

Dr. Carrara is the founder and Editor-in-Chief of BioNanoScience and Associate Editor of the IEEE TRANSACTIONS ON BIOMEDICAL CIRCUITS AND SYSTEMS and of the IEEE SENSORS JOURNAL.

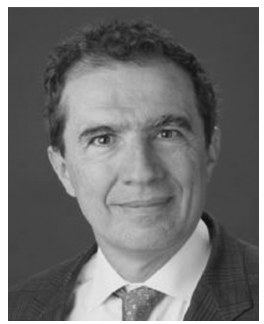

Giovanni De Micheli (F'94) received the nuclear engineer degree from Politecnico di Milano, Italy, in 1979, and the M.S. and Ph.D. degrees in electrical engineering and computer science from the University of California, Berkeley, in 1980 and 1983 , respectively.

$\mathrm{He}$ is Professor and Director of the Institute of Electrical Engineering and of the Integrated Systems Centre at École Polytechnique Fédérale de Lausanne (EPFL), Lausanne, Switzerland. He is Program Leader of the Nano-Tera.ch program. Previously, he was Professor of Electrical Engineering at Stanford University, Stanford, CA. His research interests include several aspects of design technologies for integrated circuits and systems, such as synthesis for emerging technologies, networks on chips and 3D integration. He is also interested in heterogeneous platform design including electrical components and biosensors, as well as in data processing of biomedical information. He authored Synthesis and Optimization of Digital Circuits (McGraw-Hill, 1994) and has coauthored and/or coedited eight other books as well as over 500 technical articles.

Dr. De Micheli is a Fellow of ACM and a member of the Academia Europaea and the Scientific Advisory Board of IMEC and STMicroelectronics. He is the recipient of the 2012 IEEE/CAS Mac Van Valkenburg award for contributions to theory, practice and experimentation in design methods and tools and of the 2003 IEEE Emanuel Piore Award for contributions to computer-aided synthesis of digital systems. He received the Golden Jubilee Medal for outstanding contributions to the IEEE CAS Society in 2000, the D. Pederson Award for the best paper in the IEEE TRANSACTIONS ON COMPUTER-AIDED DESIGN OF INTEGRATED CiRCUITS AND Systems in 1987, and several Best Paper Awards, including DAC (1983 and 1993), DATE (2005) and Nanoarch (2010 and 2012). He has served IEEE in several capacities, namely: Division 1 Director (2008-2009), cofounder and President Elect of the IEEE Council on EDA (2005-2007), President of the IEEE CAS Society (2003), Editor-in-Chief of the IEEE TRANSACTIONS ON COMPUTER-AIDED DESIGN OF INTEGRATED CiRCUITS AND SYSTEMS (1987-2001). He has been Chair of several conferences, including DATE (2010), pHealth (2006), VLSI SOC (2006), DAC (2000), and ICCD (1989). 